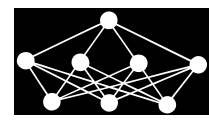

\title{
TESTING MSW TYPE OF NONLINEARITY USING AUTOCOPULAS
}

\author{
Jana Lenčuchová
}

\begin{abstract}
Inspired by Rakonczai et al. [8], we use autocopulas for the testing of linearity against Markov-switching type of nonlinearity and remaining nonlinearity. They applied this autocopula approach to testing heteroscedasticity in AR-ARCH model. Given a strictly stationary time series $Y_{t}$, a $k$-lag autocopula is a bivariate joint distribution function of the random vector $\left(Y_{t}, Y_{t-k}\right)$. Our contribution is in extending the idea to test the linearity against Markov-switching type of nonlinearity and remaining nonlinearity $[5,6]$ in order to avoid classical, time-consuming tests.
\end{abstract}

Key words: Autocopulas, MSW model, testing linearity against nonlinearity, testing remaining nonlinearity

Received: July 15, 2012

Revised and accepted: November 25, 2012

\section{Introduction}

It is convenient to test linearity against nonlinearity of a given time series before using nonlinear models. The aim is to apply simpler linear models prior to complex nonlinear models whenever possible. This step is a part of the procedure for nonlinear modeling proposed by Granger [3]:

1. Select an appropriate linear model $\operatorname{AR}(q)$ for the given time series $(q$ is a model order).

2. Test the linearity against the nonlinearity. If the null hypothesis about linearity is rejected, the step 3 will follow.

3. Estimate the parameters of the selected nonlinear model.

4. Check the appropriateness of the selected model by diagnostic tests (autocorrelation tests, a heteroskedasticity test and a test for remaining nonlinearity).

5. Modify the model if required.

* Jana Lenčuchová

Slovak University of Technology in Bratislava, E-mail: lencuchova@math.sk 
6. Use the model for the description of examined time series and for prediction purposes.

As shown in many applications, the linear model is not often sufficient because the examined time series shows a nonlinear character. Such event is typical for economic and financial time series. In this paper, we focus on one particular type of nonlinear model, so-called Markov-switching model (hereafter MSW model) from the class of regime-switching models with the regimes determined by unobservable variables. It means that occurrence of each regime in time is followed by some probability.

The MSW models were first introduced by Goldfeld and Quandt [2] in 1973 but their popularity has been rising since the publication by Hamilton [4] in 1990. He specified the stochastic process of switches between regimes as a first-order Markov process.

This paper analyzes the issues of testing in step 2 and partially in step 4 in the already mentioned procedure. There are standard LR tests at disposal. But there are some obstacles for their use, such as enormous computation requirements, problems with likelihood function and nuisance parameters unidentified under the null hypotheses. All these are described in details in the paper by Hansen [6].

These difficulties brought us to the idea of employing the autocopula approach invented by Rakonczai et al. [8]. They use the autocopula tool to distinguish between AR and AR-ARCH model in the sense of detecting conditional heteroscedasticity.

Autocopulas are copulas which are related to a time series. Defining arbitrary lag, the autocopula is the copula of the original and lagged time series in the binary case. The advantage of their use is the ability of describing the interdependence structure by taking the nonlinear relationship into account, unlike the autocorrelation function.

This concept seems to be suitable for our necessity to tell apart some linear and nonlinear MSW model or two MSW models with different number of regimes.

This paper is organized as follows. After introduction, brief theory about copulas, autocopulas and copula goodness-of-fit test is summarized in the Sections 2 and 3. Section 4 describes the MSW models, classical approach to testing linearity against the MSW-type of nonlinearity and remaining nonlinearity with the proposed alternatives by using of autocopulas. Further, there is a simulation study and conclusion is made.

\section{Copulas and Autocopulas}

Firstly, a bivariate copula function is defined [7].

Definition 1 A copula is a function $C$ from $[0,1] \times[0,1]$ to $[0,1]$ with the following properties:

(i) boundedness - for every $u, v$ in $[0,1]$

$$
C(u, 0)=C(0, v)=0
$$


and

$$
C(u, 1)=u \quad \text { and } \quad C(1, v)=v ;
$$

(ii) 2-increasing property - for every $u_{1}, u_{2}, v_{1}, v_{2}$ in $[0,1]$ such that $u_{1} \leq u_{2}$ and $v_{1} \leq v_{2}$,

$$
C\left(u_{2}, v_{2}\right)-C\left(u_{1}, v_{2}\right)-C\left(u_{2}, v_{1}\right)+C\left(u_{1}, v_{1}\right) \geq 0 .
$$

Sklar [9] proved in 1959 that $H(x, y)=C(F(x), G(y))$, where $H$ is the joint distribution function of a random vector $(X, Y)$ with marginals $F$ and $G$. If the marginals are continuous, the copula is unique. Thus, the copula function has other important interpretation as the joint distribution function.

Definition 2 Given a strictly stationary time series $Y_{t}$ and $l \in \mathbb{Z}^{+}$, the l-lag autocopula $C_{Y, l}$ is the copula of the bivariate random vector $\left(Y_{t}, Y_{t-l}\right)$. The l-lag autocopulas as the function of the lag $l$ give the autocopula function.

A large amount of copula families has been defined so far. One very wellknown is called Archimedean class of copulas (see examples in Tab. I), which is characterized by the so-called generator function. This generator function $\phi(t)$ is defined on $[0,1]$ mapping to $[0, \infty]$ and it is convex, decreasing with condition $\phi(1)=0$. Such copula is defined by

$$
C(u, v)=\phi^{[-1]}(\phi(u)+\phi(v))
$$

for all $u, v \in[0,1]$, where $\phi^{[-1]}:[0, \infty] \rightarrow[0,1]$ is a pseudo-inverse of the generator. The formal definition of the pseudo-inverse of the generator is

$$
\phi^{[-1]}(t)= \begin{cases}\phi^{-1}(t) & 0 \leq t \leq \phi(0) \\ 0 & \phi(0) \leq t \leq \infty\end{cases}
$$

The pseudo-inverse with generator gives an identity

$$
\phi^{[-1]}(\phi(t))=t
$$

\begin{tabular}{llll} 
Name & $C_{\theta}(u, v)$ & $\phi_{\theta}(t)$ & Parameter \\
\hline Gumbel & $e^{-\left((-\ln u)^{\theta}+(-\ln v)^{\theta}\right)^{\frac{1}{\theta}}}$ & $(-\log t)^{\theta}$ & $\theta \in[1, \infty)$ \\
Frank & $-\frac{1}{\theta} \ln \left(1+\frac{\left(e^{-\theta u}-1\right)\left(e^{-\theta v}-1\right)}{\left(e^{-\theta}-1\right)}\right)$ & $-\log \frac{e^{-\theta t}-1}{e^{-\theta}-1}$ & $\theta \in(-\infty, 0) \bigcup(0, \infty)$ \\
Clayton & $\left(u^{-\theta}+v^{-\theta}-1\right)^{\frac{-1}{\theta}}$ & $t^{-\theta}-1$ & $\theta \in(0, \infty)$ \\
Joe & $1-\left((1-u)^{\theta}+(1-v)^{\theta}-(1-x)^{\theta}(1-v)^{\theta}\right)^{1 / \theta}$ & $-\log \left(1-(1-t)^{\theta}\right)$ & $\theta \in[1, \infty)$ \\
Ali-Mikhail-Haq & $\frac{(u v)}{1-\theta(1-u)(1-v)}$ & $\frac{1-\theta}{e^{t}-\theta}$ & $\theta \in[-1,1)$
\end{tabular}

Tab. I Some families of Archimedean copulas. 


\section{Copula Goodness-of-Fit (GoF) Test}

The fit of copulas should be measured to be able to compare different models. This GoF test is based on Kendall's transform of the joint distribution function, which reduces multivariate problem to one dimension as follows

$$
\mathcal{K}(t)=\operatorname{Pr}(H(X, Y) \leq t)=\operatorname{Pr}(C(F(X), G(Y)) \leq t)=\operatorname{Pr}(C(u, v) \leq t) .
$$

The empirical version of $\mathcal{K}$ is calculated by the formula

$$
\mathcal{K}_{n}(t)=\frac{1}{n} \sum_{i=1}^{n} \mathbf{1}\left(E_{\text {in }} \leq t\right)
$$

where

$$
E_{i n}=\frac{1}{n} \sum_{j=1}^{n} \mathbf{1}\left(u_{j} \leq u_{i}, v_{j} \leq v_{i}\right)
$$

is so-called empirical copula and $\left\{\left(u_{i}, v_{i}\right), i \in\{l+1, \ldots, n\}\right\}$ is a sample. We use Cramér-von Mises type test statistics

$$
S_{n}=\int_{0}^{1}\left(\kappa_{n}(t)\right)^{2} d t
$$

based on Kendall's process $\kappa_{n}(t)=\sqrt{n}\left(\mathcal{K}\left(\theta_{n}, t\right)-\mathcal{K}_{n}(t)\right)$ for checking the match of the "theoretical" $\mathcal{K}(t)$ and its empirical version. These test statistics are investigated in Genest et al. [1] in detail. Numerical approximation of (9) with modifications to capture tail behavior as suggested also in [9] can be seen in Tab. II, where $\left(t_{i}\right)_{i=1}^{m}$ is an appropriately fine division of the interval $(0,1)$.

\begin{tabular}{ll}
\hline Focused Regions & Test statistics \\
\hline Global & $S_{1}=\frac{1}{m} \sum_{t_{i} \in[0+\epsilon, 1-\epsilon]}\left(\mathcal{K}\left(\theta_{n}, t_{i}\right)-\mathcal{K}_{n}\left(t_{i}\right)\right)^{2}$ \\
Upper Tail & $S_{2}=\frac{1}{m} \sum_{t_{i} \in[0+\epsilon, 1-\epsilon]} \frac{\left(\mathcal{K}\left(\theta_{n}, t_{i}\right)-\mathcal{K}_{n}\left(t_{i}\right)\right)^{2}}{1-\mathcal{K}\left(\theta_{n}, t_{i}\right)}$ \\
Lower Tail & $S_{3}=\frac{1}{m} \sum_{t_{i} \in[0+\epsilon, 1-\epsilon]} \frac{\left(\mathcal{K}\left(\theta_{n}, t_{i}\right)-\mathcal{K}_{n}\left(t_{i}\right)\right)^{2}}{\mathcal{K}\left(\theta_{n}, t_{i}\right)}$ \\
Lower and Upper Tail & $S_{4}=\frac{1}{m} \sum_{t_{i} \in[0+\epsilon, 1-\epsilon]} \frac{\left(\mathcal{K}\left(\theta_{n}, t_{i}\right)-\mathcal{K}_{n}\left(t_{i}\right)\right)^{2}}{\mathcal{K}\left(\theta_{n}, t_{i}\right)\left(1-\mathcal{K}\left(\theta_{n}, t_{i}\right)\right)}$ \\
\hline
\end{tabular}

Tab. II Numerical approximation of Cramér-von Mises type test statistics (9).

\section{Testing Linearity Against MSW Type of Nonlinearity}

Let us use the basic autoregressive MSW model, which assumes $\operatorname{AR}(q)$ model in each regime

$$
y_{t}=\varphi_{0, s_{t}}+\varphi_{1, s_{t}} y_{t-1}+\ldots+\varphi_{q, s_{t}} y_{t-q}+\epsilon_{t}, \quad s_{t}=1,2, \ldots, N
$$


for $t=q+1, \ldots, T$, where $T$ is the length of time series, $\left\{\epsilon_{t}\right\}$ is iid process and $\epsilon_{t} \sim$ $N\left(0, \sigma_{\epsilon}^{2}\right)$. Notice that the value of an observation $y_{t}$ in autoregressive coefficients is dependent on variable $s_{t}$, which is random and unobservable. It determines the so-called "regime" or "state". In case of $N$ possible regimes, such random variable can attain values from the set $\{1,2, \ldots, N\}$. The way of changing regimes is defined by the process $\left\{s_{t}\right\}$, which is specified as a first-order Markov process (Hamilton 1990 [4]). It holds that

$$
\operatorname{Pr}\left(s_{t}=j \mid s_{t-1}=i, s_{t-2}=k, \ldots\right)=\operatorname{Pr}\left(s_{t}=j \mid s_{t-1}=i\right)=p_{i j},
$$

where $i, j, k=1, \ldots, N$. Transition probability $p_{i j}$ means that the probability of the regime $j$ in time $t$ is conditional only on the previous regime $i$ in time $t-1$. So-called transition matrix $\mathbf{P}=\left(p_{i j}\right)_{i, j=1}^{N}$ is created with these probabilities. Obviously, it is satisfied that $\sum_{j=1}^{N} p_{i j}=1$ for all $i=1, \ldots, N$. The process is also homogenous because it is time-invariant on time.

This type of model is widely used in economics and finance for time series with nonlinear dynamic behavior such as Gross Domestic Product (considering regime of growth, recession and stagnation). The regimes are determined by unobservable variable, thus we know the probability with which the process is in a certain state.

The next subsections deal with the classical approach to the test of linearity against the MSW type of nonlinearity and with the new proposed test using autocopulas.

\subsection{Classical approach}

Classical testing linearity against the MSW-type of nonlinearity is computed via likelihood ratio test, where the null hypothesis is represented by a linear model, which in terms of the 2-regime MSW model can be written by the equality of autoregressive coefficients in both regimes as $H_{0}: \boldsymbol{\varphi}_{\mathbf{1}}=\boldsymbol{\varphi}_{\mathbf{2}}$ against the alternative $H_{1}: \varphi_{i, 1} \neq \varphi_{i, 2}$ for at least one $i \in\{0,1,2, \ldots, q\}$.

Likelihood ratio test statistic is difference between loglikelihood functions of a MSW model and a corresponding linear equivalent

$$
L=L_{M S W}-L_{A R}
$$

Unfortunately, this test statistic has no standard probability distribution (Hansen 1992 [6]), which means that it cannot be expressed by analytical formula. Actually, here we encounter the problem of non-defining of the nuisance parameters such as transition probabilities under the null hypothesis. Simulation can resolve it, but it requires "days" spent by computations for a bigger amount of tested time series. Such simulation experiment consists of generating a large number of artificial time series $y^{*}$ under the null hypothesis, then of estimating parameters of both models for each generated time series and calculating simulated critical values from the test statistic (12). All has to be done for each time series and for each model order $q$, respectively. 


\subsection{Goodness-of-fit test for autocopulas}

Unlike the classical approach, the test using autocopulas is completely different. We try to find out whether this test is capable to catch the MSW type of nonlinearity comparing with linearity represented by an autocopula fitted to linear autoregressive model series.

The fit of autocopula models at different lags $l$ has to be checked after estimating a given time series model. Formally, the null hypothesis of the GoF test $\mathcal{H}_{0}: C_{y_{t}, l} \in$ $\mathcal{C}_{0, l}=\left\{C_{\theta, l}: \theta \in \Theta\right\}$ for any lag $l \geq 1$.

Let us have the time series $y_{1}, y_{2}, \ldots, y_{T}$. Then, a $l$-lag sample pairs of observations is $\left\{\left(y_{t}, y_{t-l}\right), t \in\{l+1, \ldots, T\}\right\}$. But the sample for an autocopula needs to be adapted to the copula assumption for iid observations. Thus, only every sth pair is taken from the set of pairs below to destroy serial dependence between them. Dependence between dimensions should only remain. The new thinned set of time points is denoted as $\mathcal{T}=\{l+1, s+l+1,2 s+l+1, \ldots, r s+l+1 \leq T\}$, where $|\mathcal{T}|=r+1$ is the new thinned sample size, $\left\{\left(y_{t}, y_{t-l}\right), t \in \mathcal{T}\right\}$. Value $s$ should be large enough but we still need to have a sufficient number of pairs for testing. Then, one has to map margins to the unit interval by means of the empirical univariate distribution function with probability integral transformation (PIT) defined by

$$
\{\underbrace{\left\{\left(\frac{\sum_{j \in \mathcal{T}} \mathbf{1}\left(y_{j} \leq y_{i}\right)}{|\mathcal{T}|+1}\right.\right.}_{u_{i}}, \underbrace{\left.\frac{\sum_{j \in \mathcal{T}} \mathbf{1}\left(y_{j-l} \leq y_{i-l}\right)}{|\mathcal{T}|+1}\right)}_{v_{i}}: i \in \mathcal{T}\} .
$$

Now we got the sample $\left\{\left(u_{i}, v_{i}\right), i \in \mathcal{T}\right\}$ with uniformly distributed margins. The testing procedure consists of:

1. Simulate AR time series with the size $n=10000$ for 500 times.

2. Obtain their $l$-lag autocopula sample from the series thinned by $s=10$.

3. Calculate the test statistics $S_{i, j}, i=1, \ldots, 4$ and $j=1, \ldots, 500$ and choose the 0,95 quantiles as critical values $Q_{i ; 0,95}, i=1, \ldots, 4$.

4. Simulate the MSW model time series with the size $n=10000$ for 500 times.

5. Obtain their $l$-lag autocopula sample from the series thinned by $s=10$.

6. Calculate the test statistics $S_{i, j}, i=1, \ldots, 4, j=1, \ldots, 500$ and reject $H_{0}$ whenever $S_{i, j}>Q_{i ; 0,95}$.

Then, we can calculate a rejection rate of the null hypothesis referring to a linear model against the alternative of a 2-regime MSW model.

\section{Testing Remaining Nonlinearity}

The idea above is also applied to the test of remaining nonlinearity. The null hypothesis is represented by a 2-regime MSW (MSW2) model against the alternative of a 3-regime MSW (MSW3) model. The test is carried out via a likelihood ratio with the following test statistic: 


$$
L=L_{M S W 3}-L_{M S W 2} .
$$

The same story about the enormous time-consumption test holds for the standard test for the remaining nonlinearity.

The testing procedure using autocopulas is the same but instead of the sample simulated from an AR model we need a sample from a 2-regime MSW model and instead of the model with 2-regimes it is required to simulate time series sample from a 3-regime MSW model.

\section{Simulation Experiments}

Two main experiments were done. The first one for testing linearity against MSW type of nonlinearity and the second one for the testing remaining nonlinearity.

\subsection{Setting values}

We need three time series models: a linear autoregressive model, a 2-regime MSW model and a 3-regime MSW model. The starting input values are set as follows:

- linear autoregressive model of order $q=1$ with $\varphi_{0}=0 ; \varphi_{1}=0,5$ (hereafter $\operatorname{AR}(0,5))$

- 2-regime MSW model of the same order in both regimes with $\varphi_{10}=0 ; \varphi_{11}=$ 0,$7 ; \varphi_{20}=0 ; \varphi_{21}=0,3$ and the transition matrix $\mathbf{P}=\{\{0,6 ; 0,7\},\{0,4 ; 0,3\}\}$ (hereafter MSW2 $(0,7 ; 0,3)$ for the transition matrix given below if not specified otherwise)

- 3-regime MSW model of the same order in all three regimes with $\varphi_{10}=0$; $\varphi_{11}=0,7 ; \varphi_{20}=0 ; \varphi_{21}=0,3 ; \varphi_{30}=0 ; \varphi_{31}=0,1$ and the transition matrix $\mathbf{P}=\{\{0,2 ; 0,8 ; 0,4\},\{0,3 ; 0,1 ; 0,2\},\{0,5 ; 0,1 ; 0,4\}\}$ (hereafter MSW3 $(0,7 ; 0,3 ; 0,1)$ for the transition matrix given below if not specified otherwise)).

The random innovations $\epsilon_{t}$ are drawn from $N(0,1)$ and the same are injected to both models. Significance level is set to $\alpha=0,05$; the sample size $n=10000$ and the thinning parameter $s=10$. Copula parameters were estimated by the minimization of $L_{2}$ norm distance between an exact and empirical copula.

Also, other model settings were investigated, thus some of parameters such as autoregressive parameters and transition matrices were altered (see Tabs. IV and $\mathrm{V})$.

To detect time series lagged interdependence, these well-known copulas were employed to look for the copula closest to the one generated by MSW model: Gumbel, Frank, Clayton, Joe, Ali-Mikhail-Haq, Farlie-Gumbel-Morgenstern, and Gaussian copula. 


\subsection{Results - testing linearity against MSW-type of nonlinearity using autocopulas}

By this test we try to identify a deviation from linearity especially to nonlinearity of the MSW-type. Tab. III offers results of the ability to distinguish between the linear model representing the null hypothesis and the 2-regime MSW model in the alternative. The Frank copula achieved the highest ability to reveal such deviaton in $47,2 \%$ of all cases for 1-lag autocopula for the test statistics S3 (lower tail weighted) and S4 (lower and upper tail weigthed). Such medium probability results are not too encouraging. Thus, we continue to analyze an impact of changing parameters in the 2-regime MSW model. First, the value of the autoregressive coefficient in the second regime is varied and then the transition matrix.

\begin{tabular}{|c|c|c|c|c|}
\hline \multicolumn{5}{|c|}{ AR vs. MSW2 model } \\
\hline Copula & TS & $\mathrm{l}=1$ & $\mathrm{l}=2$ & $\mathrm{l}=3$ \\
\hline \multirow{4}{*}{ GUMBEL } & S1 & $6,4 \%$ & $7,6 \%$ & $6,4 \%$ \\
\hline & S2 & $5,8 \%$ & $5,6 \%$ & $6,0 \%$ \\
\hline & S3 & $6,0 \%$ & $9,2 \%$ & $5,2 \%$ \\
\hline & S4 & $6,4 \%$ & $7,0 \%$ & $5,8 \%$ \\
\hline \multirow{4}{*}{ FRANK } & S1 & $32,0 \%$ & $36,0 \%$ & $25,8 \%$ \\
\hline & $\mathrm{S} 2$ & $44,4 \%$ & $32,8 \%$ & $27,2 \%$ \\
\hline & S3 & $47,2 \%$ & $37,2 \%$ & $24,0 \%$ \\
\hline & S4 & $47,2 \%$ & $36,2 \%$ & $25,8 \%$ \\
\hline \multirow{4}{*}{ CLAYTON } & S1 & $8,5 \%$ & $5,5 \%$ & $9,0 \%$ \\
\hline & $\mathrm{S} 2$ & $11,5 \%$ & $5,5 \%$ & $12,0 \%$ \\
\hline & S3 & $7,5 \%$ & $10,5 \%$ & $8,5 \%$ \\
\hline & S4 & $12,5 \%$ & $8,5 \%$ & $11,0 \%$ \\
\hline \multirow{4}{*}{ GAUSSIAN } & S1 & $27,5 \%$ & $38,5 \%$ & $11,0 \%$ \\
\hline & $\mathrm{S} 2$ & $28,5 \%$ & $28,5 \%$ & $12,5 \%$ \\
\hline & S3 & $33,0 \%$ & $36,5 \%$ & $14,5 \%$ \\
\hline & S4 & $33,5 \%$ & $38,5 \%$ & $12,0 \%$ \\
\hline \multirow{4}{*}{ JOE } & S1 & $11,8 \%$ & $16,0 \%$ & $12,4 \%$ \\
\hline & $\mathrm{S} 2$ & $7,2 \%$ & $13,2 \%$ & $13,4 \%$ \\
\hline & S3 & $16,4 \%$ & $25,6 \%$ & $15,8 \%$ \\
\hline & S4 & $12,0 \%$ & $18,6 \%$ & $16,0 \%$ \\
\hline \multirow{4}{*}{ FGM } & S1 & $33,2 \%$ & $6,8 \%$ & $7,5 \%$ \\
\hline & $\mathrm{S} 2$ & $33,4 \%$ & $9,0 \%$ & $13,0 \%$ \\
\hline & S3 & $29,6 \%$ & $9,6 \%$ & $10,5 \%$ \\
\hline & S4 & $39,4 \%$ & $10,2 \%$ & $10,0 \%$ \\
\hline \multirow{4}{*}{ AMH } & S1 & $15,0 \%$ & $3,6 \%$ & $1,8 \%$ \\
\hline & S2 & $19,5 \%$ & $3,8 \%$ & $1,4 \%$ \\
\hline & S3 & $9,5 \%$ & $2,8 \%$ & $1,6 \%$ \\
\hline & $\mathrm{S} 4$ & $19,0 \%$ & $4,2 \%$ & $1,6 \%$ \\
\hline
\end{tabular}

Tab. III Rejection rates of the null hypothesis of the $A R(0,5)$ model if the true model is $M S W(0,7 ; 0,3) ; \mathbf{P}=\{\{0,6 ; 0,7\},\{0,4 ; 0,3\}\}$. 
Notice that provided the results in Tab. IV, taking the Frank copula, the rejection rates of the null hypothesis are growing with higher value of autoregressive coefficient $\varphi_{21}$, that is the ability to reveal nonlinear interdependences looks up. It is caused by the increased measure of dependence between variables. Tab. IV also includes Kendall's correlation coefficient for each case. The correlation coefficients of the tested time series should be similar sufficiently because the autocopula with stronger dependence structure could outperform the weaker one. For the sample generated from $\operatorname{AR}(0,5)$ model, Kendall's $\tau=0,333$.

\begin{tabular}{lrrrrr}
\hline$\varphi_{21}$ & 0,1 & 0,3 & 0,5 & 0,7 & 0,9 \\
\hline S1 & $0 \%$ & $32 \%$ & $97 \%$ & $100 \%$ & $100 \%$ \\
S2 & $3 \%$ & $44 \%$ & $98 \%$ & $100 \%$ & $100 \%$ \\
S3 & $2 \%$ & $47 \%$ & $99 \%$ & $100 \%$ & $100 \%$ \\
S4 & $2 \%$ & $47 \%$ & $99 \%$ & $100 \%$ & $100 \%$ \\
\hline Kendall's $\tau$ & 0,310 & 0,368 & 0,430 & 0,494 & 0,560 \\
\hline
\end{tabular}

Tab. IV Rejection rates of the null hypothesis of the $A R(0,5)$ model if the true model is $\left.M S W\left(0,7 ; \varphi_{21}\right) ; \mathbf{P}=\{\{0,6 ; 0,7\},\{0,4 ; 0,3\}\}\right)$, lag $l=1$, number of simulations $=200$ and using Frank copula.

Interesting results appeared in case of changing values in transition matrix (Tab. V). It seems that the length of regime persistence in the process has serious effect on the rejection rate. We defined new ones, where there is expressed different "willingness" of a process to stay in particular regime. First two transition matrices represent "lazy" process. It is highly probable that the process stays in the same regime. Here we can observe improved ability to distinguish between the MSW model time series with long periods in both regimes and the AR model. As we increased probability $p_{21}$ and left $p_{11}$ relatively high (columns 5 and 6 in Tab. V), we got really significant rejection rates of the null hypothesis under alternative model being valid. The test failed totally when regimes swapped very often.

\begin{tabular}{lrrrrrrr}
\hline $\mathbf{P}$ & $\left(\begin{array}{c}0,90,2 \\
0,10,8\end{array}\right)$ & $\left(\begin{array}{l}0,950,1 \\
0,050,9\end{array}\right)$ & $\left(\begin{array}{c}0,70,5 \\
0,30,5\end{array}\right)$ & $\left(\begin{array}{l}0,60,7 \\
0,40,3\end{array}\right)$ & $\left(\begin{array}{ll}0,8 & 0,7 \\
0,20,3\end{array}\right)$ & $\left(\begin{array}{l}0,950,7 \\
0,050,3\end{array}\right)$ & $\left(\begin{array}{l}0,10,8 \\
0,90,2\end{array}\right)$ \\
\hline S1 & $70,5 \%$ & $80,5 \%$ & $38,5 \%$ & $32,0 \%$ & $94,5 \%$ & $100 \%$ & $1,0 \%$ \\
S2 & $79,0 \%$ & $83,0 \%$ & $45,5 \%$ & $44,4 \%$ & $96,0 \%$ & $100 \%$ & $2,5 \%$ \\
S3 & $85,0 \%$ & $89,0 \%$ & $51,5 \%$ & $47,2 \%$ & $96,0 \%$ & $100 \%$ & $2,0 \%$ \\
S4 & $82,5 \%$ & $87,0 \%$ & $51,0 \%$ & $47,2 \%$ & $98,0 \%$ & $100 \%$ & $1,5 \%$ \\
\hline
\end{tabular}

Tab. V Rejection rates of the null hypothesis of the $A R(0,5)$ model if the true model is the $M S W(0,7 ; 0,3)$; number of simulations $=200$ and using Frank copula.

\subsection{Results - testing remaining nonlinearity}

The theory of nonlinear modeling says that after the parameter fitting of each nonlinear model (in our case the 2-regime MSW model), one should use diagnostic testing for checking model adequacy. Actually we need to know if 2 regimes are fully sufficient or if there is a space for adding the next regime. Therefore, the 2-regime with 3-regime MSW model is tested by using autocopulas taking Frank copula, as 
proposed in the subsection above in case of testing linearity against the MSW type of nonlinearity. Tab. VI shows the poor nearly zero ability to differentiate between models with 2 and 3 regimes for employed copulas and given input values.

\begin{tabular}{|c|c|c|c|c|}
\hline \multicolumn{5}{|c|}{ MSW2 vs. MSW3 model } \\
\hline & S1 & $4,4 \%$ & $5,5 \%$ & $6,5 \%$ \\
\hline \multirow[t]{3}{*}{ GUMBEL } & S2 & $6,0 \%$ & $19,5 \%$ & $9,5 \%$ \\
\hline & $\mathrm{S} 3$ & $0,4 \%$ & $1,0 \%$ & $7,0 \%$ \\
\hline & $\mathrm{S} 4$ & $5,4 \%$ & $8,0 \%$ & $8,0 \%$ \\
\hline \multirow{4}{*}{ FRANK } & S1 & $0 \%$ & $0 \%$ & $0 \%$ \\
\hline & $\mathrm{S} 2$ & $0 \%$ & $0 \%$ & $0 \%$ \\
\hline & S3 & $0 \%$ & $0 \%$ & $0 \%$ \\
\hline & $\mathrm{S} 4$ & $0 \%$ & $0 \%$ & $0 \%$ \\
\hline \multirow{4}{*}{ CLAYTON } & S1 & $1,0 \%$ & $1,5 \%$ & $0,5 \%$ \\
\hline & $\mathrm{S} 2$ & $0 \%$ & $0 \%$ & $0 \%$ \\
\hline & S3 & $1,0 \%$ & $0 \%$ & $0,1 \%$ \\
\hline & $\mathrm{S} 4$ & $0 \%$ & $0,7 \%$ & $0,5 \%$ \\
\hline \multirow{4}{*}{ GAUSSIAN } & S1 & $0 \%$ & $0 \%$ & $0 \%$ \\
\hline & S2 & $0 \%$ & $0 \%$ & $0 \%$ \\
\hline & $\mathrm{S} 3$ & $0 \%$ & $0 \%$ & $0 \%$ \\
\hline & $\mathrm{S} 4$ & $0 \%$ & $0 \%$ & $0 \%$ \\
\hline \multirow{4}{*}{ JOE } & $\mathrm{S} 1$ & $0,5 \%$ & $0 \%$ & $0 \%$ \\
\hline & $\mathrm{S} 2$ & $0 \%$ & $0 \%$ & $0 \%$ \\
\hline & S3 & $0,5 \%$ & $0 \%$ & $0 \%$ \\
\hline & $\mathrm{S} 4$ & $0 \%$ & $0 \%$ & $0 \%$ \\
\hline \multirow{4}{*}{ FGM } & S1 & $0 \%$ & $3,0 \%$ & $12,0 \%$ \\
\hline & $\mathrm{S} 2$ & $0 \%$ & $1,0 \%$ & $4,0 \%$ \\
\hline & S3 & $0 \%$ & $1,0 \%$ & $9,0 \%$ \\
\hline & $\mathrm{S} 4$ & $0 \%$ & $2,0 \%$ & $9,0 \%$ \\
\hline \multirow{4}{*}{$\mathrm{AMH}$} & S1 & $0 \%$ & $0,6 \%$ & $3,4 \%$ \\
\hline & $\mathrm{S} 2$ & $0 \%$ & $0,2 \%$ & $3,2 \%$ \\
\hline & S3 & $0 \%$ & $0,8 \%$ & $5,6 \%$ \\
\hline & $\mathrm{S} 4$ & $0 \%$ & $0,4 \%$ & $3,6 \%$ \\
\hline
\end{tabular}

Tab. VI Rejection rates of the null hypothesis of the $M S W(0,7 ; 0,3) ; \mathbf{P}=$ $\{\{0,6 ; 0,7\},\{0,4 ; 0,3\}\}$ if the true model is $M S W(0,7 ; 0,3 ; 0,1) ; \mathbf{P}=\{\{0,2 ; 0,8$; $0,4\},\{0,3 ; 0,1 ; 0,2\},\{0,5 ; 0,1 ; 0,4\}\}$.

Also, the impact of the parameter changing was considered. When the autoregressive parameter in the third regime $\varphi_{31}$ alternates, the similar story occured as in the case of testing the linear model against the 2-regime MSW model. The rejection rate increased with the higher autoregressive parameter in the third regime, see Tab. VII. The possible reason for getting such low rejection rates in Tab. VI is smaller Kendall's $\tau=0,241$ for the 3-regime MSW model generating sample in the alternative hypothesis. Thus, autocopula from the 3 -regime model was somehow overwhelmed by the one from the 2-regime model with stronger dependence structure. Concerned for various transition matrices, let us look at Tab. VIII. Su- 
perior ability to reveal remaining nonlinearity is only in the transition matrix with extremely high transition probabilities from one to the same regime $\left(p_{11}, p_{22}, p_{33}\right)$. Other investigated cases were not so successful or not successful at all.

\begin{tabular}{lrrrrr}
\hline$\varphi_{21}$ & 0,1 & 0,3 & 0,5 & 0,7 & 0,9 \\
\hline S1 & $0 \%$ & $0 \%$ & $1,8 \%$ & $52,4 \%$ & $99,6 \%$ \\
S2 & $0 \%$ & $0 \%$ & $2,2 \%$ & $55,2 \%$ & $99,6 \%$ \\
S3 & $0 \%$ & $0 \%$ & $1,2 \%$ & $51,6 \%$ & $99,6 \%$ \\
S4 & $0 \%$ & $0 \%$ & $1,6 \%$ & $57,0 \%$ & $100 \%$ \\
\hline Kendall's $\tau$ & 0,241 & 0,298 & 0,356 & 0,416 & 0,478 \\
\hline
\end{tabular}

Tab. VII Rejection rates of the null hypothesis of the $M S W(0,7 ; 0,3) ; \mathbf{P}=$ $\{\{0,6 ; 0,7\},\{0,4 ; 0,3\}\}$ model if the true model is $M S W\left(0,7 ; 0,3 ; \varphi_{31}\right) ; \mathbf{P}=$ $\{\{0,2 ; 0,8 ; 0,4\},\{0,3 ; 0,1 ; 0,2\},\{0,5 ; 0,1 ; 0,4\}\})$, lag $l=1$, number of simulations $=200$ and using Frank copula.

\begin{tabular}{|c|c|c|c|c|}
\hline $\mathbf{P}$ & $\left(\begin{array}{lll}0,7 & 0,1 & 0,1 \\
0,2 & 0,8 & 0,2 \\
0,1 & 0,1 & 0,7\end{array}\right)$ & $\left(\begin{array}{lll}0,7 & 0,1 & 0,3 \\
0,2 & 0,8 & 0,3 \\
0,1 & 0,1 & 0,4\end{array}\right)$ & $\left(\begin{array}{ccc}0,9 & 0,01 & 0,02 \\
0,05 & 0,95 & 0,04 \\
0,05 & 0,04 & 0,94\end{array}\right)$ & $\left(\begin{array}{ccc}0,99 & 0,01 & 0,0005 \\
0,005 & 0,98 & 0,0005 \\
0,005 & 0,01 & 0,999\end{array}\right)$ \\
\hline S1 & $0 \%$ & $44,0 \%$ & $46,5 \%$ & $95,5 \%$ \\
\hline S2 & $0 \%$ & $37,5 \%$ & $44,5 \%$ & $96,5 \%$ \\
\hline S3 & $0 \%$ & $48,5 \%$ & $36,0 \%$ & $88,5 \%$ \\
\hline $\mathrm{S} 4$ & $0 \%$ & $43,5 \%$ & $47,5 \%$ & $96,5 \%$ \\
\hline
\end{tabular}

Tab. VIII Rejection rates of the null hypothesis of the $M S W(0,7 ; 0,3)$ model if the true model is the $M S W(0,7 ; 0,3 ; 0,5)$; number of simulations $=200$ and using Frank copula.

\section{Conclusion}

The new approach using autocopulas to testing linearity against the MSW type of nonlinearity and testing remaining nonlinerity was analyzed. There are classical tests at disposal but they are really time-consuming. We were inspired by Rakonczai et al. [8], they used autocopulas for testing heteroskedasticity in the AR-ARCH model. Since the MSW model does not generate an autocopula from any well-known copula families, we fitted several of them and determined the closest one. We found out that parameter settings of the time series model affect the dependence structure of autocopula significantly.

Concerning test of linearity against the MSW-type of nonlinearity, in several cases, some copulas were able to recognize the presence of regimes in time series. The Frank copula denoted the best results, mainly in cases of regimes with long periods in one or both regimes.

In case of testing remaining nonlinearity, none of the tested copulas was able to detect adequately necessity of the third regime in the model for given input values. The partial success occured when the parameters were varied.

The setting of input values should be considered in further research, best according to real models. As shown in the section with results, reasonable parameter 
configuration for models is crucial. Moreover, our results are also limited by the selection of copulas we were using, and maybe the "right" one was not examined. But such simulations are very time-consuming, thus we had to choose only a couple of them.

There remain more experiments to be performed before recommending the copula approach as an alternative to classical test for linearity. A methodology to eliminate the effect of dependence strength needs to be developed. Also comparison of computational time consumption and power of the test between the new and the classical approach is needed.

\section{Acknowledgement}

This work was supported by the grants APVV No. LPP-0111-09 and VEGA $1 / 0143 / 11$.

\section{References}

[1] Genest C., Quessy J. F., Rémillard B.: Local efficiency of a Cramér-von Mises test of independence, Journal of Multivariate Analysis 97, 2006, pp. 274-294.

[2] Goldfeld S. M., Quandt R. E.: A Markov Model for Switching Regressions. Journal of Econometrics, 1, 1973, pp. 3-16.

[3] Granger C. W. J.: Strategies for modelling nonlinear time-series relationships, The Economic Record, 69, 1993, pp. 233-238.

[4] Hamilton J. D.: A New Approach to the Economic Analysis of Nonstationary Time Series and the Business Cycle, Econometrica, 57, 2, 1990, pp. 357-384.

[5] Hamilton J. D.: Time series analysis, Princeton University Press, Princeton, 1994.

[6] Hansen B. E.: The likelihood ratio test under nonstandard assumptions: testing the Markov switching model of GNP, Journal of Applied Econometrics, 7, 1992, pp. 61-82.

[7] Nelsen R. B.: An Introduction to Copulas, 2nd ed. John Wiley \& Sons, 2006.

[8] Rakonczai P., Márkus L., Zempléni A.: Autocopulas: investigating the interdependence structure of stationary time series, Methodology and Computing in Applied Probability, 14, 1, 2012, pp. 149-167.

[9] Sklar A.: Fonctions de répartition à n dimensions et leurs marges, Publ. Inst. Stat. Univ. Paris, 8, 1959, pp. 229-231. 